\title{
A Pattern Recognition for Group Abnormal Behaviors Based on Markov Random Fields Energy
}

\author{
Li Zuojin, Chen Liukui \\ College of Electrical and Information \\ Engineering \\ Chongqing University of Science and \\ Technology \\ Chongqing, China, 401331 \\ cqustlzj@sina.cn
}

\author{
Ren Zhiyong \\ Department of Communication \\ Chongqing College of Electronic \\ Engineering \\ Chongqing, China,401331 \\ cqulzj@gmail.com
}

\author{
Sreenivas Sremath Tirumala \\ Department of Computing \\ Faculty of Creative Industries and \\ Business \\ Unitec of Institute and Technology \\ Auckland, Newzealand,1142 \\ zli2@unitec.ac.nz
}

\begin{abstract}
Group abnormal behaviors often occur abruptly under video surveillance, thus bringing serious consequences. How to recognize these behaviors correctly has always been the difficulty in research on intelligence video surveillance. This paper is based on the basic theory of Markov Random Fields to extract the features of those in video images, so as to recognize the group abnormal behaviors under video surveillance. Experiments show that this method can well reflect the real situation at the spot.
\end{abstract}

Keywords-markov model; pattern recognition; abnormal behaviors

\section{INTRODUCTION}

In recent years, group abnormal behaviors often occur in public, which may cause very serious consequences in some cases. Therefore, how to notice these behaviors promptly has become an urgent need for safeguard in key public places. Video surveillance system, as an important precaution technology, has been widely used in public safeguard [1-3]. The ever increasing cameras and coverage, however, can not ensure prompt recognition of abnormal behaviors from a large volume of video information by means of manual attendance. For this reason, quick recognition of abnormal behaviors based on powerful information processing ability of computers and real-time analysis of surveillance video is an important but difficult point in the research on intelligence video surveillance system.

At present, the major way of detecting the video abnormal behaviors is extracting the optical flow field features in the information flow of video, which are used to express the energy of pixels in video. However, as the scenes under surveillance are often complex, the computation of the energy of optical flow fields may be interfered with background information, making the feature extraction quite difficult, and thus the recognition rate is reduced. Markov random field is a random distribution model, based on MRF model and Bayes theory. MRF model connects the uncertain description with priori knowledge and, with the images under observation, determines the objective function of the partition problems with the optimal criterion in the statistical decision and estimation theory. Then it works out the largest possible distribution meeting these conditions or consumption function, and consequently transforms the partition problems into optimization problems. Hence, MRF model has been widely applied in image processing [4-7].

\section{MRF IMAGE MODEL}

Definition: two-dimension neighborhood system [8]

Let, $\quad M=\left\{m_{i j}(i, j) \in N \times N, m_{i j} \subset N \times N\right\}$ and for any $(i, j) \in N \times N, m_{i j} \subset N \times N$,

1) $(i, j) \notin m_{i j}$

2) and if $(k, l) \notin m_{i j}$, then $(i, j) \notin m_{k l}$

Therefore, $m_{i j}$ is a neighborhood of $(i, j)$ and $M$ is one neighborhood system in $N \times N$.

\section{Definition: cliques}

Let $M$ be one neighborhood system in $N \times N$ and $C$ is a subset in $N \times N$ relevant to $M$. And if $C$ is composed of a single pixel (a point on the plane), or

if $(i, j) \neq(m, n), \quad(i, j) \in c, \quad(m, n) \in c \quad$, then $(i, j) \in m_{k l}$

Therefore, $C$ is referred to be a clique relevant to $M$. All cliques on $M$ are marked as $C$. Figure 1 shows an example of a two-order neighborhood. 

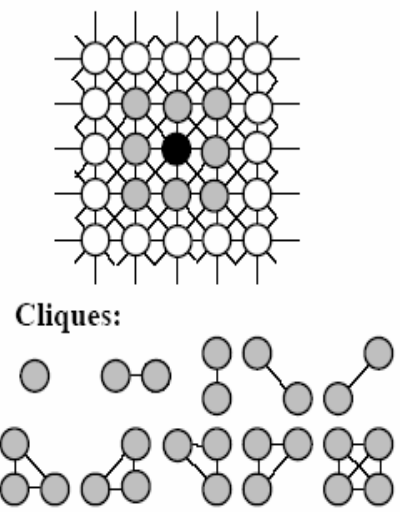

Fig. 1. Two-order neighborhood system on the plane

\section{Definition: two-dimension MRF}

Let $X=\left\{X_{i j}, i, j \in N\right\}$ be a random field in the twodimension square $N \times N(N=\{1,2,3 \ldots \ldots . . r\}) \quad$, and $M=\left\{m_{i j} \quad(i, j) \in N \times N, m_{i j} \subset N \times N\right\} \quad$ are neighborhood systems. Then if for all $(i, j) \in N \times N$, $P\left\{X_{i j} \mid X_{k l}, \quad(i, j) \in N \times N,(i, j) \neq(k, l)\right\}=P\left\{X_{i j}, \quad(k, l) \in m_{i j}\right\}$ , we can refer to $X$ as a two-dimension Markov random field (MRF) relevant to the neighborhood system $M$.

\section{CALCULATION METHOD OF VIDEO ENERGY}

Now we take the video frame from a surveillance camera as a MRF model, and the random variables $x$ and $y$ becomes the scale value of the pixel $s, t(t \in N(s))$, i.e. the gray value of the image. According to the Section 2, the energy function of the $n$-th frame of the video images is defined as:

$$
E_{M}(n)=\sum_{s}\left|x_{s}(n)-y_{s}\right|+\lambda \sum_{s} \sum_{t \in N(s)}\left|x_{s}(n)-x_{t}(n)\right|(1)
$$

In this equation (1), $E_{M}(n)$ is the data energy, $x_{s}(n)$ is the gray value of the pixel $s$ in the n-th frame of the video image, and $y_{s}(n)$ is that on the corresponding position in the background image. And $\lambda$ is the important parameter for calculating the group energy, as shown in the following equation:

$$
\lambda=\frac{\sigma_{\psi}^{2}}{\sigma_{\phi}^{2}}
$$

In the equation (2), $\sigma_{\psi}^{2}$ is the variance of the variable $\left|x_{x}(n)-y_{s}(n)\right|$ and $\sigma_{\phi}^{2}$ is that of $\sum_{t \in N(s)}\left|x_{s}(n)-x_{t}(n)\right|$.

\section{EXPERIMENTS AND CONCLUSIONS}

To prove the feasibility of Markov random field energy in recognition of abnormal behaviors, we choose two scenes with typical group activities as the objects for experiment, shown in Figure 2. Figure 2 (a) shows a gathering of fewer people than those in (b). To reflect the group activities more accurately, we set five degrees of intensity, namely stationary, gentle movement, slow movement, quick movement and violent movement. In experiment, colors ranging from blue to red are used to represent the five degrees of intensity as shown in Figure 3. The results tell us that Figure (b) has larger red area than that of (a), suggesting more violent movement occur in the corresponding area in scene (b). This well reflects the features of group abnormal behaviors. Similarly, Figure 3 (a) has larger blue area than that of (b), demonstrating that in these areas there are no people or no movement, which is consistent with the real situation.

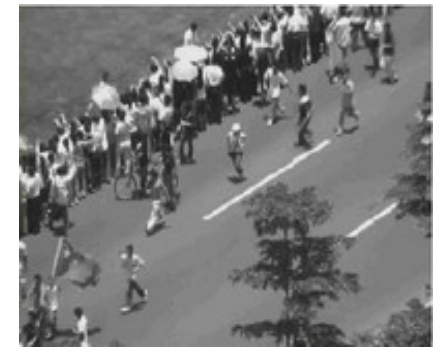

(a)

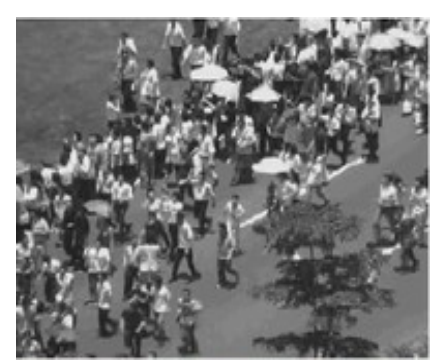

(b)
Fig. 2. the video monitoring scene. the deferent Group abnormal in (a) and (b)

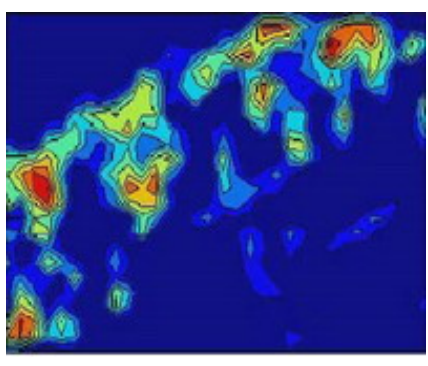

(a)

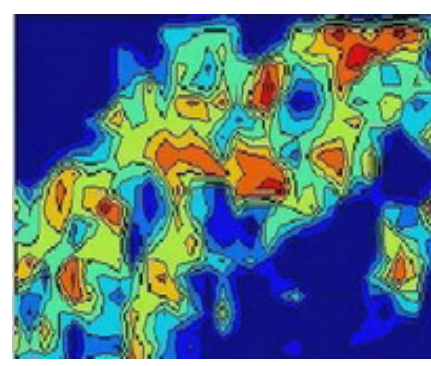

(b)
Fig. 3. The MRF energy directional distribution for fig 2 (a) and (b) respectively

It has been proved that we can use energy distribution figure to represent the position and area with dense crowds of people and appraise their movement. When the group movement become intense and the red area become larger, early warning can be given, so as to avoid the abnormal behaviors like congestion, hustling, or chaos. To summarize, the video energy features based on Markov random field proposed in this paper can correctly recognize group abnormal 
=behaviors, which provides some theoretical support to the intelligence video surveillance system.

\section{ACKNOWLEDGMENT}

This work was supported financially by Scientific and Technological Research Program of Chongqing (Grant No. KJ131423, No. KJ131422 and KJ132206), Doctor Special Project from Chongqing University of Science and Technology (Grant No. CK2011B05 and CK2011B09) and Natural Science Foundation of Chongqing (Grant No. cstcjjA40041).

\section{REFERENCES}

[1] Wu Xuzhan, Li Gaoli, Research and Application Development of the Video Surveillance System [J]. Journal of Salt and Chemical Industry, 2013, 42(8):6-9.
[2] Zhao Penghui,Yan Ying, Analysis of the conception and characteristics of video monitoring system [J], Electronic Design Engineering, 2013, 21(14):188-120.

[3] Xie Yi, Bao Kejin, Research and Realization on Face Detection in Intelligent Video Surveillance [J], Computer Measurement and contol, 2013, 21(11):2921-2913.

[4] Li Xuchao, Zhu Shanan, A Survey of the Markov Random Field Method for Image Segmentaition [J], Journal of Image and Graphics, 2007, 12(5):789-798.

[5] Li Qing, Shi Xiaokai, A Hidden Markov Template Model for $\mathrm{R}$ ecognizing Human Activities from Videos [J], Journal of WUT (Information and Management Engineering), 2013, 35(6):789-793.

[6] Li Hongsheng, Xu Hongzhang, Image' s denoising research based on hidden Markov parameter model [J], Laser and Infrared,2013, 43(10):1184-1187.

[7] He Feiyue, Tian Zheng, Efficient Segmentation of SAR Image Using Markov Random Field Models with Edge Penalties and an Adaptive Weighting Parameter [J], Acta Optica Sinica, 2013, 33(8):1-8.

[8] T. Simchony, R. Chellappa, and Z. Lichtenstein. Relaxation algorithms for MAPestimation of gray-level images with multiplicative noise. IEEE Trans. On Information Theory, 1990. 36(3): 608-613. 